\title{
Instances statutaires et organisation administrative du Collège de France
}

$\mathrm{Au} 1^{\mathrm{er}}$ décembre 2018

\section{OpenEdition \\ Journals}

Édition électronique

URL : https://journals.openedition.org/annuaire-cdf/16544

DOI : 10.4000/annuaire-cdf.16544

ISBN : 978-2-7226-0572-5

ISSN : 2109-9227

Éditeur

Collège de France

\section{Édition imprimée}

Date de publication : 30 décembre 2020

Pagination : 775-786

ISBN : 978-2-7226-0516-9

ISSN : 0069-5580

Référence électronique

"Instances statutaires et organisation administrative du Collège de France », L'annuaire du Collège de France [En ligne], 118 | 2020, mis en ligne le 01 avril 2021, consulté le 22 août 2022. URL : http://

journals.openedition.org/annuaire-cdf/16544; DOI : https://doi.org/10.4000/annuaire-cdf.16544 


\title{
V. INSTANCES STATUTAIRES ET ORGANISATION ADMINISTRATIVE DU COLLÈGE DE FRANCE
}

\author{
au $1^{\text {er }}$ décembre 2018
}

\begin{abstract}
ASSEMBLÉE DU COLLÈGE DE FRANCE
Membres avec voix délibérative : les professeurs titulaires en exercice.

Participent avec voix consultative : le directeur général des services et l'agent comptable. Les professeurs honoraires, les professeurs sur chaire annuelle, les professeurs invités sur une base annuelle ou pluriannuelle peuvent être conviés par l'administrateur.

Président : Alain Prochiantz, administrateur du Collège de France.

Vice-président : Thomas RÖMER.

Secrétaire : Françoise COMBE.
\end{abstract}

\section{BUREAU DU COLLÈGE DE FRANCE}

Alain PROCHIANTZ, administrateur, président.

Thomas RÖMER, vice-président.

Marc FONTECAVE, secrétaire.

Dominique CHARPIN, professeur.

Françoise COMBES, professeure.

Antoine COMPAGNON, professeur.

Jean DALIBARD, professeur.

Hugues DE THÉ, professeur.

Pierre-Michel MENGER, professeur.

Jean-Marie TARASCON, professeur.

Marylène MESTON DE REN, directrice générale des services.

CONSEIL D'ÉTABLISSEMENT

Président :

Alain PROCHIANTZ, administrateur. 
Professeurs :

Patrick BOUCHERON.

Anne CHENG.

Jean DALIBARD.

Hugues DE THÉ.

Jean-Luc FoURNET.

François HÉRAN.

Henry LAURENS.

Thomas RÖMER.

Clément SANCHEZ.

Représentants des personnels :

Sous-collège B2 :

Sylvie RIMSKI.

Sous-collège C1 :

Guillaume LEBAILLY.

Virginie MONCEAU.

Sous-collège C2 :

Lionel MARTI (CNRS).

Christophe NICOLLE (CNRS).

Clément SAYRIN (UPMC).

Marie-Émilie TERRET (Inserm).

Sous-collège D1 :

Khalid BENHAMMAD.

Claire GUTTINGER.

Gordana JOACHIM.

Stéphane LLALIAS.

Sous-collège D2 :

Rodrigo ALVEAR-PEREZ (Inserm).

Nicole BRAURE (Inserm).

Philippe SIMON (CNRS).

Personnalités extérieures:

1. Représentants d'organismes scientifiques :

Véronique DeBISSCHOP, déléguée régionale, CNRS.

Jean-Frédéric GERBEAU, directeur général délégué à la science, Inria.

Gilles BLOCH, président-directeur général, Inserm.

2. Représentant des activités économiques:

Gaël Dupont, vice-président, directeur général délégué, Cholet Dupont.

Membres de droit avec voix consultative :

Marylène MESTON DE REN, directrice générale des services.

Philippe LEZER, agent comptable. 


\section{BUREAU DU CONSEIL D’ÉTABLISSEMENT}

Marylène MESTON DE REN.

Claire GUTTINGER.

Lionel MARTI.

\section{ADMINISTRATEUR}

Alain ProchIANTZ, [C.

Assistante de direction: $\quad$ Patrizia DELU, poste 12.48 .

\section{DIRECTION GÉNÉRALE DES SERVICES}

Directrice générale des services: Marylène MESTON DE REN (赵), poste 11.02.

Assistancelgestion :

Rosemary PORPIGLIA, poste 11.04 .

Céline SAHIN, poste 11.24.

Chargée d'affaires juridiques : $\quad$ Rahma RIBARDIÈRE, poste 14.12 .

Pôle Achat public :

Emma TORRÈs-FONTAINE, poste 13.03.

Nathan MARCILLAT, poste 11.38.

Marion FolLENFANT, poste 10.73.

Projets transversaux: $\quad$ Mathilde BERTOU, poste 11.04.

Barbara LITZLER, poste 14.16.

Mission valorisation :

Anne DUMAS, poste 11.79 .

Keltoum HAROUAT, poste 13.13.

Ganthiry N'DIAYE, poste 11.44.

\section{DIRECTION DES AFFAIRES CULTURELLES}

ET RELATIONS EXTÉRIEURES

Directrice :

Florence TERRASSE-RIOU, poste 11.01.

Gestion budgétaire et secrétariat : Véronique-Anne MicHALOWSKI, poste 11.07.

Enseignement, organisation

des colloques:

Sophie BENITTA, poste 11.45 .

Véronique-Anne MichaLOWSKI, poste 11.45.

\section{Pôle Publications}

Responsable :

Éditrices :

Suivi juridique, administratif et logistique :
Céline VAUTRIN, poste 13.45 .

Emmanuelle FLEURY, poste 10.37.

Anne-Laure GÉNIN, poste 11.16.

Martine TORREGROSSA, poste 12.47 . 


\section{DIRECTION DES RELATIONS INTERNATIONALES, DU DÉVELOPPEMENT ET DES PARTENARIATS}

Directeur :

Relations avec la presse:

Relations internationales:

Gestion du service et des chaires annuelles:

Gestion des chaires internationales et missions à l'étranger des professeurs :
Raynald BELAY, poste 10.40 .

Guillaume KASPERSKI, poste 12.72 .

Céline SURPRENANT, poste 12.82 .

Frédéric DOYEN, poste 17.33.

Véronique GudIN, poste 14.72.

\section{PÔLE WEB ET MULTIMÉDIA}

Responsable :

Marco CUCCHI, poste 10.08 .

Assistante, suivi des contrats des captations, partenariat

France Culture :

Sandrine EтchetTo, poste 13.02.

Site internet :

Claire JEANNEQUin, poste 10.29.

David ADJEMIAN, poste 10.18 .

Marie-Catherine MANEZ, poste 14.53.

Réseaux sociaux :

David ADJEMIAN, poste 10.18.

Infographie :

Mona VALLERY, poste 10.67 .

Production vidéo :

Marco CUCCHI, poste 10.08 .

\section{DIRECTION DES RESSOURCES HUMAINES}

Directrice :

Chargée des ressources

humaines:

Assistance, secrétariat :
Stéphanie ALEXANDRE, poste 11.30.

Maud BABAU-LAMBERT, poste 12.43 .

Kyle Michardy, poste 11.23.

\section{Pôle PILOTAGe}

\section{Emplois, affaires générales et recrutements}

Responsable :

Éric VAN ZANDT, poste 11.84 .

Assistante :

Fabienne JANÈS-TONIOLO, poste 11.21.

\section{Masse salariale et contrôle interne}

Responsable :

Loréa FÉRET, poste 11.26.

Assistante :

Karine MAILLARD, poste 10.95 .

Pôle GESTION DES PERSONNELS TITULAIRES ET CONTRACTUElS

Responsable : 
Enseignants-chercheurs, personnels extérieurs :

Fatine PIÉQUET, poste 12.51 .

Contractuels, titres de séjour: $\quad$ Tiziana MANICONE, poste 10.58.

Personnels BIATSS et titulaires ITRF :

Aline DUROCHER, poste 11.90.

Titulaires AENES, bibliothèques

et personnels contractuels : $\quad$ Sonya JoB, poste 16.31 .

Gestion des rémunérations

du budget propre :

Françoise CRÉPIN, poste 11.27.

\section{Pôle ACCOMPAGNEMENT PROFESSIONNEL}

Responsable :

Assistante administrative :

Cellule handicap :
Estelle DÉSIR, poste 11.61.

Marie-Claudine AH-PET, poste 11.28.

Estelle DÉSIR, poste 11.61.

Pôle de MÉdecine de PRÉvention

$\begin{array}{ll}\text { Médecins : } & \begin{array}{l}\text { Dr François-Xavier CALLIES. } \\ \text { Dr Juliette PAGEZY. }\end{array} \\ \text { Infirmière : } & \text { Marie DUMAS, poste 11.51. } \\ \text { Secrétariat : } & \text { Samia HADDAD, poste 11.69. } \\ \text { Assistante en gestion } & \\ \text { administrative : } & \text { Carol AHÉHÉHINNOU, poste 10.48. }\end{array}$

Pôle D'ACTION SOCIALE

Gestionnaire :

Fabienne JANÈS-TONIOLO, poste 11.23.

Assistante sociale:

Sandrine CORDARY, poste 11.23.

\section{DIRECTION DES AFFAIRES BUDGÉTAIRES ET FINANCIÈRES}

Directrice :

Sophie VIACROZE, poste 11.58.

Assistante :

Audrey NisCOISE, poste 15.24.

Pôle Pilotage et budget

Responsable :

Maryama ABBASSI, poste 13.15.

Pôle EXÉcution budGÉTAIRE, QUALITÉ ET MAîTRISE DES RISQUES

Responsable :

Bureau des dépenses:
Juliette MAILLARD, poste 11.34.

Yanne GNADOU, poste 10.10.

Francesca ANDRIAMANANTSARA, poste 13.04.

Nadia BELMEHBOUL, poste 11.31.

Kevin BAHNAM, poste 10.34.

Amaury PELlut, poste 11.20. 
Bureau des recettes,

des conventions hors recherche

et de la comptabilité analytique: Fatoumata DIAKHABY, poste 13.15.

\section{AGENCE COMPTABLE}

Agent comptable :

Philippe LEZER, poste 14.73.

Fondé de pouvoir :

Étienne MACHEFER, poste 11.37.

Béatrice MARCHAIS, poste 13.92.

Renata MARTIN, poste 10.64.

Axelle PelliCIARI, poste 11.87.

\section{DIRECTION DES SYSTĖMES D'INFORMATION}

Directeur :

Secrétariat :

\section{MisSION SÉCURITÉ DU SI}

RSSI, correspondant CNIL et MESR :

RSSI suppléant :

\section{Mission Pilotage}

Intranet et communication interne :

Isabelle SARDELLA, poste 14.78.

\section{ProjeTs}

SI de gestion :

Gilles JANDEAU, poste 14.00 .

SI financier et SI décisionnel: $\quad$ Lionel VINCENTI, poste 11.80.

Projets de parcs informatiques : Julie ChamPagNe, poste 14.14 .

\section{INFRASTRUCTURES}

Téléphonie et annuaires:

Administration des systèmes :

Gordana JOAQUIM, poste 15.98 .

Cyrille GHESQUIÈRE, poste 12.20.

Daniel GERHARD, poste 15.25.

\section{BUREAUTIQUe}

Responsable :

Gilles JANDEAU, poste 14.00.

Intervenants :
Patrick GASPALDY, poste 10.71 .

Franck ADAM, poste 14.14.

Aurélien AGNERAY, poste 10.55. 


\section{Audiovisuel}

Responsable régie audiovisuelle: Gilles DEBONNE, poste 10.35.

Régisseurs :

Gilles DEBOnNE, poste 10.35.

Thibaud MASSET, poste 19.74 .

Bruno PAGESSE, poste 19.74 .

Francisco VALDERRAMA, poste 19.74 .

Henry CARRASQUERO, poste 19.74 .

Service photographique: $\quad$ Patrick IMBERT, poste 11.39.

\section{DIRECTION DU PATRIMOINE IMMOBILIER}

Directeur :

Bovis LiBÉREAU, poste 11.32.

Secrétariat :

Caroline BRUNO, poste 11.22.

Chargée de coordination

administrative et financière: $\quad$ Rachida MEZGHENNA, poste 11.36.

\section{SERVICE DES TRAVAUX IMMOBILIERS}

Responsable du service:

Béatrice MATÉOS, poste 11.50.

Conduite d'opérations

immobilières, pilotage :

Béatrice MATÉOS, poste 11.50.

Plans, relevés, assistance

en suivi de travaux:

Olivier PopotTe, poste 10.69.

Chargée d'opération

immobilière :

Jeanne Lo RE, poste 10.39.

\section{SERVICE ENTRETIEN-MAINTENANCE}

Responsable du service : Pierre MOREAU, poste 11.66.

Coordinateurs techniques de sites

Marcelin Berthelot

Stéphane LALLIAS, poste 13.28.

Ulm et Nogent-sur-Marne :

Jean MANGin D'HeRMANTIN, poste 11.68 .

\section{Secteurs}

Entretien des bâtiments

par une entreprise extérieure: $\quad$ Stéphane LALLIAS, poste 13.28.

Chauffage, ventilation,

climatisation :

Yassin DIAF, poste 11.35.

Électricité :

Jean MANGIN D'HeRmantin, poste 11.68.

\section{Chefs d'ateliers}

Électricité :

Jean Mangin D'HeRmantin, poste 11.68 .

Plomberie, menuiserie-serrurerie, peinture:

Laurent LEICHNIG, poste 11.48 . 


\section{Ateliers}

Menuiserie-serrurerie :

Plomberie-sanitaires :

Peinture, revêtement sol:

Électricité :
Christian JULIEN, poste 11.53.

Coraline CORBET, poste 11.53.

Mustapha GHANES, poste 11.57.

Roger BORNE, poste 11.54.

Samir AOUABED, poste 11.55.

Soupramaniane PARAMESVARIN, poste 11.55.

Damien CHAUMEIL, poste 11.55.

\section{PÔLE HYGIÈNE-SÉCURITÉ}

Responsable :

Adjoint :

Assistante administrative :
Xavier BlondEAU, poste 11.18.

Benjamin SAPPEY, poste 11.86.

Marie-Claudine АH-PET, poste 11.28.

\section{DIRECTION DE LA LOGISTIQUE}

Directeur :

Sébastien BERROYER, poste 11.05/11.13.

Télécopie : 01.44.27.11.17.

Adjoint :

Stéphane GRELLIER, poste 11.14/11.13.

\section{GESTION DES SITES, ENTRETIEN DES LOCAUX}

Responsable :

Suivi de l'entretien des locaux: Olivier CALMO, poste 10.88 .

Entretien des locaux:

ACHAT, FACTURATION

Responsable :

Gestion financière,

suivi des contrats :
Lydie LESUR, poste 12.75 .

Stéphane GRELLIER, poste 11.14.

Marie-Line LHOMME, poste 19.14.

Hanisu DEBRETSION, poste 19.14.

Muriel MATHEN, poste 19.14.

Neela WIJESINGHA, poste 19.14.

Jean-Jacques NOTON

Bertrand CRÉPIN, poste 10.60 .

\section{GESTION DES MARCHÉS, CARTE MULTISERVICES}

Responsable :

Chauffeur :

Intendance :

Contrôles d'accès, carte multiservices :
Florian AUDISIO, poste 12.80 .

Romain PETGES, poste 10.88 .

Philippe VASSEUR, poste 11.13.

Jean-Luc ATANASIO, poste 11.47. 


\section{ACCUEIL - SÉCURITÉ, MANUTENTION, REPROGRAPHIE}

Responsable :

Accueil :

Manutention :

Reprographie :

Sécurité :

Loge Marcelin-Berthelot :

Loge Ulm :

Loge Belle-Gabrielle :

\section{COURRIER, LIVRAISON}

Responsable :

Courrier :

Livraisons, magasins :
David Fleurman, poste 11.03.

Aurélie PALIN, poste 11.47.

Gérard ElOIRE, poste 19.13. Madani MoudJENIBA, poste 19.13.

Antony LEFÈVRE, poste 19.13 .

Abdella KHALDI, poste 14.68.

Sandanam RocK, poste 11.47.

Étienne et Ombline SMITH, poste 11.11.

Bernard et Martine MÉGUEIL, poste 11.72.

Gilbert et Brigitte RIGOLE, poste 11.63.

Soy KORN-GENTILINI, poste 15.97.

Marie-Line MORVANY, poste 15.97.

Hervé SAINT-AURET, poste 15.82 / 19.28 .

Rémy THÈZE, poste 15.82 / 19.28.

\section{DIRECTION DES RÉSEAUX ET DES PARTENARIATS DOCUMENTAIRES}

Directrice :

Site Marcelin Berthelot Site La Belle Gabrielle

Adjoint à la directrice :

Informatique documentaire :

Archives:

Ressources en ligne, bibliométrie formation des usagers, base Zotero :

Magasinier, prêt entre bibliothèques $(P E B)$ :

Secrétariat :

Atelier de reliure :
Anne CHATELLIER, conservatrice générale, poste 17.92 . poste 13.57.

Guillaume LEBAILLY, conservateur, poste 17.17.

Carmen Alemany, poste 13.68.

Claire GuTTINGER, poste 13.63 .

Gianna SERGI, poste 13.49.

Philippe MichOU-SAUCET, poste 17.96.

Antoine HUSZAR, poste 10.36 . Thomas ESTRIER, poste 17.94.

Cécile Andrienne ThymBA, poste 17.88 .

Valérie PAUTRAT, poste 17.24.

Christine KHALIL, poste 18.97.

\section{BIBLIOTHĖQUE PATRIMONIALE}

Responsable :

Gestion des revues, reliure:

Catalogage :
Carmen Alemany, poste 13.68.

Ronan LE GUEN, poste 17.94.

Flavien PellenC, poste 17.96. 
Accueil, traitement des collections :

Secrétariat :

Responsable:

Adjoint, gestion des fonds scientifiques et audiovisuels, Salamandre :

Traitement des fonds d'archives: Frédérique PAILLADES, poste 13.49.

Chargée de mission scientifique: Laure LEVEILLÉ, poste 14.05.

Photothécaire :
Angélique CHIOROZAS, poste 14.05.

Thomas ESTRIER, poste 17.94.

Cécile Andrienne THYMBA, poste 17.88.
Antoine HuSZAR, poste 10.36.

\section{ARCHIVES}

Claire GutTINGER, poste 13.63.

Christophe LaBAUne, poste 10.52 .

\section{BIBLIOTHÈQUES SPÉCIALISÉES}

\section{INSTITUT DES CIVILISATIONS}

\section{Pôle Proche-Orient AnCIEN}

Directeur :

\section{Égyptologie}

Directeur :

Bibliothèque et archives :

\section{Proche-Orient ancien}

Directeur :

\section{Assyriologie \\ Bibliothèque :}

\section{Études ouest-sémitiques}

Bibliothèque :
Thomas RÖMER, professeur au Collège de France poste 10.50 .

Nicolas GrIMAL, professeur au Collège de France poste 10.46 .

Elsa RICKAL, responsable poste 10.47.

Lucie HIGEL, poste 10.47.

Cécile Bernal, poste 10.47 .

Thomas RÖMER, professeur au Collège de France poste 10.50 .

Loraine MARCHEIX, responsable, poste 10.43 . Mina THEROND, poste 15.31.

Loraine MARCHEIX, responsable, poste 10.51.

Catherine RAYNAL, poste 10.51 .

Nicolas MARINBAYONA, poste 10.51. 


\section{Pôle Monde MÉditerRanéEn ANCIEN, MÉdiéval ET MODERnE}

Directeur :

François DÉROCHE, professeur au Collège de France poste 10.26 .

\section{Études arabes, turques et islamiques}

Directeur :

Assistante :

Bibliothèque :

\section{Études byzantines}

Directeur :

UMR 8167 :

Bibliothèque :

\section{Pôle EXTRÊME-ORIENT}

Directeur :

Service général :

Parc informatique :

\section{Études chinoises}

Directrice :

Publications :

Bibliothèque :

\section{Études coréennes}

Directeur :

Bibliothèque :
Nicolas VATIN, directeur d'études à l'EPHE poste 17.80 .

Elisabetta BORROMEO, poste 17.81.

Driss MEKOUAR, responsable, poste 17.87. Elif BECAN, poste 17.87 .

Jean-Luc FouRNET, professeur au Collège de France poste, 17.72 .

Vincent DEROCHE, poste 17.73.

Guillaume LEBAILLY, poste 17.17.

Nina IAMANIDZE, poste 17.97.

Morgane MoRIZUR, poste 15.48.

Konstantin VETOCHNIKOV, poste 17.97.

Jean Noël RoBERT, professeur au Collège de France, poste 18.13.

Sophie BOSSER, poste 11.94.

Georges-Alexandre LE CORRONC, poste 10.98 .

Jean-François RABORIO, poste 18.11.

Marianne BUJARD, directrice de recherches à l'EPHE, poste 10.06 .

Isabelle ANG, maître de conférences, poste 18.27.

Delphine SPICQ, responsable, poste 18.08.

Émilie DEVIENNE, poste 18.08.

Lucienne DURIEZ, poste 18.09.

Wing Fong LEUNG, poste 18.79.

Esther LIN, poste 18.94.

Denise SMITH, poste 18.69.

Alain DELISSEN, directeur d'études à l'EHESS, poste 18.32 .

Mi-Sug No-GENETIOT, responsable, poste 18.14. Kyung Min SHIM, poste 18.14. 


\section{Études indiennes}

Directeur :

Bibliothèque :

Publications :

\section{Études japonaises}

Directeur :

Bibliothèque :

\section{Études tibétaines}

Directrice :

Bibliothèque :
Frantz GRENET, professeur au Collège de France, poste 16.13 .

Ronan MOREAU, responsable, poste 18.07.

Caroline RiberaiguA, responsable, poste 18.10.

Anabelle MUKENDI, poste 17.95.

Isabelle SZELAGOWSKI, poste 18.28.

Jean-Noël ROBERT, professeur au Collège de France, poste 18.13.

Kaoru BABA, responsable, poste 18.54. Nathalie CAZAL, responsable de la bibliothèque et en charge des publications, poste 18.06. Édouard NIERGA, poste 18.06.

PôLE ANTHROPOLOGIE SOCIALE

\section{Anthropologie sociale Bibliothèque Claude Lévi-Strauss}

Directeur :

Sous-directrice :

Gestion financière :

Bibliothèque \& Archives:
Philippe DESCOLA, professeur au Collège de France, poste 10.12.

Brigitte DERLON, poste 17.50.

Patricia RigOLE, poste 17.65 .

Sophie ASSAL, responsable, poste 17.46. Vincent DUCHAUFFOUR, poste 17.67. Sandrine LECOINTRE, poste 17.54. Marie-Christine VICKRIDGE, poste 17.64. Nicolas ADDE, poste 17.32.

\section{MEMBRE ASSOCIÉ}

\section{Société asiatique}

Télécopie : 01.44.27.11.70

Directeur :

Jean-Pierre MAHE, membre de l'Institut, poste 18.05.

Bibliothèque :
Françoise WANG-TOUTAIN, poste 18.30.

Marie PreZIosi-Boute, responsable, poste 18.30.
Caroline GYss, poste 18.05.

Amina ABUDUREHEMAN, poste 18.05. 